\title{
El espacio como elemento clave para la regulación emocional en la escuela: análisis en contextos de diversidad social y cultural
}

\author{
Fuentes Vilugrón, Gerardo Andrés \\ El espacio como elemento clave para la regulación emocional en la escuela: análisis en contextos de diversidad \\ social y cultural \\ Revista Educación, vol. 44, núm. 2, 2020 \\ Universidad de Costa Rica, Costa Rica \\ Disponible en: http://www.redalyc.org/articulo.oa?id=44062184017 \\ DOI: https://doi.org/10.15517/revedu.v44i2.39365
}

Esta obra está bajo una Licencia Creative Commons Atribución-NoComercial-SinDerivar 3.0 Internacional. 


\title{
El espacio como elemento clave para la regulación emocional en la escuela: análisis en contextos de diversidad social y cultural
}

\author{
Surroundings as a Key Element for Emotional Regulation at School: Analysis of Contexts of Social and Cultural \\ Diversity
}

Gerardo Andrés Fuentes Vilugrón

Universidad Católica de Temuco, Chile

gfuentes2019@alu.uct.cl

(iD) http://orcid.org/0000-0002-8924-7821

\author{
DOI: https://doi.org/10.15517/revedu.v44i2.39365 \\ Redalyc: http://www.redalyc.org/articulo.oa?id=44062184017
}

Recepción: 12 Diciembre 2019

Aprobación: 13 Mayo 2020

\section{Resumen:}

Los espacios y las emociones son aspectos influyentes en el desarrollo humano y en el aprendizaje, siendo el contexto educativo escolar uno de los espacios más relevantes en edades tempranas. Este ensayo aborda el problema relacionado con la educación escolar chilena y la descontextualización de contenidos producto de la invisibilización de los conocimientos y saberes indígenas en relación con el espacio y la regulación emocional. El objetivo consiste en analizar y reflexionar acerca del espacio y su influencia en la regulación de la emoción según los conocimientos occidentales e indígenas en el contexto educativo escolar caracterizado por la diversidad social y cultural. El método de este ensayo, consiste en una búsqueda sistemática, análisis e interpretación de contenidos teóricos acerca del espacio y su relación con la regulación emocional y afectiva. Al respecto, el pueblo mapuche, siendo una de las sociedades indígenas que habitan en Chile, han complejizado y otorgado el concepto de espacio, dando mayor sentido, profundidad y multidimensionalidad, en contraste con la definición tradicional. Sin embargo, la educación escolar chilena reproduce solo los saberes occidentales que afectan directamente en la identidad y autorregulación emocional de aquellos social y culturalmente diferentes.

Palabras clave: Diversidad cultural, Emociones, Espacio, Regulación emocional.

\section{Abstract:}

Surroundings and emotions are influential aspects of human development and learning. The school context is one of the most relevant surroundings for early childhood. This article addresses problems apparent in Chile's schools linked to decontextualization of content resulting from indifference to indigenous knowledge and awareness regarding surroundings and emotional regulation. The objective is to analyze and how surroundings influence emotional regulation according to western and indigenous social and cultural diversity in a school setting. Research methodology is based on a systematic search, analysis and interpretation of theoretical content about one's surroundings in relation to emotional and affective regulation. Chile's Mapuche indigenous community, has impacted the traditional notion of how school settings are understood by adding a level of complexity, depth and multidimensionality. However, this is not a consideration of Chilean schools which only to embrace western sources of knowledge, ignoring how this will impact the identity and emotional self-regulation of groups considered to be socially and culturally diverse.

KEYWORDS: Cultural Diversity, Emotions, Surroundings, Emotional Regulation.

\section{INTRODUCCIÓN}

La regulación emocional consiste en los procesos intrínsecos y extrínsecos responsables del monitoreo, evaluación y modificación de las reacciones emocionales, los cuales contribuyen de manera positiva en diferentes dominios del funcionamiento social, cultural y laboral de las personas (Medrano, Moretti, Ortiz y Pereno, 2013; Vázquez y Enríquez, 2012; Thompson, 1994). En este sentido, se ha evidenciado la existencia de diversos aspectos que influyen en la regulación emocional en la escuela, ya sea familia, ambiente, profesores y profesoras, las relaciones sociales y el espacio (García, Ortega y Rivera, 2013; Henao y García, 2009; 
Márquez-Cervantes y Gaeta-González, 2017). Es decir, la regulación de las emociones no son un aspecto únicamente personal, más bien se considera multidimensional que también incorpora el contexto social y cultural de pertenencia y ascendencia.

El espacio es una de las dimensiones influyentes en la regulación de las emociones, es considerado como parte importante de la vida humana. Puesto que su significación depende de quienes viven inmersos en él, la que deviene de la experiencia e interpretación continua de las personas establecidas en determinado lugar (Losada, 2001). Así, los espacios de mayor relevancia en el aprendizaje y desarrollo humano desde edades tempranas es el hogar y la escuela, por esta razón, la educación escolar se vuelve uno de los primeros espacios de socialización y de trasmisión de conocimientos y saberes, los cuales deben ser pertinentes al contexto y pertenencia de los niños, niñas y jóvenes.

El problema tiene relación con la educación escolar chilena y la descontextualización de los contenidos, conocimientos y saberes transmitidos, producto de las estructuras occidentales que han sido reproducidas con base en un modelo hegemónico y monocultural que ha invisibilizado los conocimientos y saberes de sociedades y culturas diferentes, como es el caso del pueblo mapuche en Chile.

El pueblo mapuche representa uno de los ocho pueblos indígenas que habitan el país, alcanzando un 79,8\% de la población indígena y el 9,9\% de la población total chilena según el censo 2017 (Instituto Nacional de Estadísticas [INE], 2017). Así, el espacio desde el contexto educativo familiar mapuche ha sido complejizado con un sentido más profundo y multidimensional. Por otro lado, el espacio desde la geografía tradicional ha sido visto solo desde su dimensión física de una manera reduccionista, ya sea la edificación de la escuela, el ambiente o los alrededores visto como un lugar o paisaje que rodea el entorno educativo escolar.

Es por ello que, la incorporación de dichos conocimientos y saberes indígenas al proceso de enseñanza y aprendizaje escolar permitiría mejorar la regulación emocional de estudiantes pertenecientes a familias indígenas y no indígenas. Considerando que ambos grupos sociales y culturales tienen una educación basada en la espacialidad, donde son incluidos los aspectos territoriales o geográficos, la historia y la espiritualidad como elementos esenciales de su educación para el desarrollo físico, cognitivo y emocional-afectivo.

\section{a. Método}

Consiste en la búsqueda y análisis sistemático de contenidos teóricos acerca de la construcción conceptual del espacio desde sus diferentes dimensiones y su relación con la regulación emocional y afectiva. Al respecto, se hizo una revisión de literatura científica y de divulgación científica que contribuyan en la profundización de las temáticas abordadas. El procedimiento y técnica utilizada para el tratamiento de los antecedentes teóricos consiste en un análisis de contenido para interpretar y develar nuevos antecedentes que permitan discutir en relación con el objeto de estudio.

\section{b. Objetivo}

El objetivo de este artículo es analizar y reflexionar sobre la dimensión espacial y su influencia en la regulación de las emociones en contexto de diversidad social y cultural presente en el aula, considerando los conocimientos y saberes mapuches en contraste con los conocimientos y saberes occidentales para desarrollar una educación pertinente que permita el reconocimiento de la diversidad en los espacios escolares.

\section{RELACIÓN ENTRE ESPACIO Y REGULACIÓN EMOCIONAL}

El ser humano tiene diferentes capacidades que se pueden dividir en procesos emocionales o afectivos, cognitivos y conductuales producto de su capacidad de sentir, pensar y actuar (Sancho y Martínez, 2011). 
En relación con esto, los afectos se conocen como los sentimientos, emociones, motivaciones, actitudes y creencias que tienen influencia directa con la cognición (Boyd, Dooley, y Odom, 2006). Además, la dimensión emocional y afectiva influye en la adquisición y desarrollo del lenguaje del alumnado, lo que según Vygotsky se convierte en uno de los componentes principales de la socialización (Calderón, 2017; Riquelme, Quilaqueo, Quintriqueo, y Loncón, 2016) que ha sido definida por Weber (1977) como "una conducta 'plural' que, por el sentido que encierra, se presenta como recíprocamente referida" (p.21). Lo que se relaciona directamente con la educación escolar, donde los niños y las niñas pasan una parte de su desarrollo infantil y adolescente (Echavarría, 2003), de tal modo que la escuela se vuelve uno de los principales escenarios de formación e interacciones social y un espacio fundamental para la transmisión de valores. No obstante, para que esto se lleve a cabo, el estudiantado debe desarrollar el sentido de pertenencia a un espacio como puede ser la escuela, la familia, la comunidad, el paisaje o entorno en el cual viven niñas, niños y jóvenes (Huerta, 2017).

El espacio ha sido subestimado por la educación y cultura occidental para la regulación de las emociones. Sin embargo, la vida del ser humano se considera emocional y espacial, dado que existe una interacción continua con los lugares, paisajes, espacios públicos, espiritualidad y tiempo, a quienes se atribuyen sus propios significados, según las propias experiencias, aspiraciones, recuerdos, tradiciones, rituales, que evocan pensamientos e ideas propias de la gente (Nogué, 2015). Así, existen diferencias en la dimensión emocional y espacial entre estudiantes pertenecientes a sociedades indígenas en relación con el grupo de estudiantes no indígenas en los contextos escolares caracterizados por su diversidad social y cultural.

\section{EL ESPACIO Y LA REGULACIÓN EMOCIONAL DESDE LOS SABERES OCCIDENTALES}

Desde la mirada eurocéntrica occidental el espacio es parte del desarrollo humano, Ruiz (1994) escribe sobre diferentes tipos de posición de la persona en el espacio, tales como posición fetal, considerada como el primer lugar donde se mueve el ser humano.

a) Espacio lactante como el apego materno que contribuye en lo físico y en lo emocional-afectivo.

b) Espacio doméstico entendido como el lugar donde el ser humano permanece durante la mayor parte de su tiempo, de modo que es trascendental en el aspecto emocional.

c) Espacio motriz definido como el límite de nuestro campo práxico y las sensaciones con el movimiento.

d) El espacio de tipo psicológico que también está sujeto al espacio físico relacionado con la formación de la personalidad, toma de decisiones, resolución de problemas, entre otros. El espacio sensorial está relacionado con el control de los sentidos.

e) Por último, el espacio afectivo tiene que ver con las emociones y el modo en que estas se regulan.

En este sentido, la escuela se vuelve un escenario el cual debe considerar todos estos aspectos espaciales que incluye el desarrollo del ser humano, para usar como herramienta en la formación del alumnado.

En el marco de la espacialidad educativa escolar, la escuela debe adaptarse constantemente a las etapas evolutivas de los y las estudiantes. Puesto que, es necesario un espacio global que contribuya al logro de los objetivos de aprendizaje que consista en una escuela para todos y todas, considerando la diversidad social y cultural presente dentro del aula, una escuela comunicativa, que contribuya al desarrollo autónomo y colectivo, donde se desarrolle el campo de la expresión y finalmente, una escuela capaz de adaptarse al momento, espacio y tiempo y que forme a las y los estudiantes para la sociedad del presente y el futuro (Ruiz, 1994).

Para esto, cabe señalar lo escrito por Losada (2001), donde hace la diferenciación entre:

a) El espacio objetual que abarca los fenómenos que son manipulables en su totalidad. 
b) El espacio cosmológico que "abarcan los fenómenos de rango de magnitud más grandes (el sol, el universo y similares)" (p.272).

c) El espacio sociogeográfico que "abarcaría todos aquellos fenómenos espaciales con que los seres humanos solo pueden confrontarse, o estar ubicados dentro de ellos, sin ser capaces de manipularlos" (p.272).

Todos estos espacios, según lo expuesto por Ruiz (1994) y por Losada (2001) son influyentes en el desarrollo humano y en su comportamiento. Por consiguiente, las experiencias vividas, tanto personales como colectivas, en los diferentes espacios, determinarán la propia significación de aquellos y aquellas que están inmersos en él.

Desde la psicología se expone otra definición del espacio, otorgándole el nombre de espacio personal o vivido y que es entendido como:

El conjunto de hechos que determinan el comportamiento de un sujeto dado en un momento determinado. Este espacio contendría al individuo mismo, los objetivos que busca realizar, los factores negativos que trata de eludir, así como las barreras que restringen y limitan sus movimientos, o bien, los caminos que debe seguir para lograr alcanzar aquello que desea (Lotito, 2009 , p. 13).

Lo anterior, deja en evidencia la mirada reduccionista con que se concibe el espacio por la geografía tradicional, definiendo el espacio como una superficie terrestre, diversidad de paisajes y distribución territorial o local dividida en regiones, países, continentes, entre otros (Vargas, 2012). Es decir, se reduce el espacio en una dimensión física y territorial que no considera a sus habitantes y el sentido que les dan a dichos lugares. No obstante, en el marco histórico de las sociedades dominantes y en el contexto del periodo de colonización, el plan de colonización europea consistía en la ocupación y conquista del territorio, y de cada habitante, por medio de la transformación al cristianismo de los pueblos indígenas con una educación cultural integral para integrarlo al estatus de cristiano por ende a la civilización que la Iglesia quería construir. De este modo, los conquistadores, criollos y criollas y las misiones debían colonizar el espacio, los cuerpos y la palabra para encauzarlas a un orden europeo y mercantilista, en que se dio paso a una censura de los aspectos históricos y cosmológicos del pueblo (Wright, 2003).

\section{EL ESPACIO Y LA REGULACIÓN EMOCIONAL DESDE LOS SABERES MAPUCHE}

En Chile, uno de los pueblos originarios es el mapuche, en la actualidad alcanzan el 10\% de la población y se dividen según su territorialidad: los Pircunche en el Norte, Los Williche en el Sur, Nagche en la precordillera de la Costa, Nagche en el valle y los Lafquenche del borde costero del océano Pacífico (Errázuriz , 2006). Una parte de la población mapuche vive en comunidades rurales, principalmente en las regiones del Maule, el Biobío, La Araucanía, Los Ríos y Los Lagos, siendo considerados como territorios ancestrales (Saavedra, 2000).

En ese contexto, ha existido una censura histórica de los saberes mapuche en el contexto educativo escolar chileno. Al respecto, Quintriqueo y Torres (2013) exponen que existen diferencias epistemológicas entre los saberes culturales mapuche y los saberes entregados en la escuela, de modo que la educación mapuche contempla la relación entre los sujetos con el espacio, ya sea natural, social, cultural y espiritual, mientras que, en la escuela son una síntesis de conocimientos científicos para el análisis de la realidad. Es decir, se desvalorizan los saberes mapuches otorgando énfasis en los conocimientos y métodos occidentales para la educación. En este sentido, "Para el mapuche, las plantas, animales, cerros y vertientes, son fuentes de conocimiento que definen formas de interacción de las personas con el medio en el que se desenvuelven" (Quintriqueo y Torres, 2013, p. 209). Puesto que, el conocimiento del medio natural se relaciona directamente con el territorio. Por otro lado, existe una vinculación de la familia mapuche con el aspecto espiritual, refiriéndose a aquellas fuerzas no visibles como posibilidad de construcción 
de conocimientos. Estos, son considerados como protectores de los espacios naturales, contribuyen a la prevención de catástrofes. El Günechen también es un referente de conocimiento, considerado como la fuerza creadora de todo, de modo que, de él provienen los conocimientos para la vida para conseguir un equilibrio entre las familias, la naturaleza y la espiritualidad.

Desde los conocimientos y saberes mapuche, Quintriqueo, Quilaqueo, Gutiérrez y Peña-Cortés (2015) identifican cinco nociones de espacio:

a) Püji, hace referencia al territorio y a la energía material y espiritual de los seres vivos.

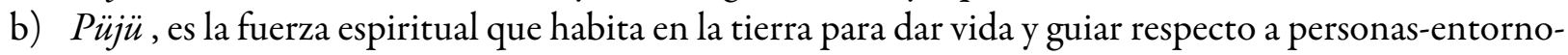
sociedad.

c) Weпu maри , es la energía superior que sostiene la tierra (espacio vertical).

d) Nag mapu, se refiere al espacio donde se genera vida física y biológica (espacio horizontal).

e) Münche mapu, hace referencia a las fuerzas que sostienen la vida bajo la tierra (espacio posterior a la vida).

Para la sociedad mapuche, el espacio es producto de una construcción histórica y social, transmitida por la memoria social en el contexto de la familia y comunidad actual (Torres y Quilaqueo, 2011). Es decir, la educación familiar mapuche se relaciona directamente con conceptos temporales y espaciales, ya sea conceptos naturales, sociales y espirituales, lo que permite a los niños, niñas y jóvenes apropiarse de su racionalidad enseñada por medio de la observación e interpretación del ciclo del sol, la luna y la naturaleza (Torres y Quilaqueo, 2011).

La naturaleza en el proceso de aprendizaje y regulación emocional de niños, niñas y jóvenes, se hace un recorrido por las geografías emocionales entendida como, el estudio que se relaciona con el territorio y su significado y el impacto en la regulación emocional y la formación de identidad (Nogué, 2014). Sin embargo, no se puede reducir la espacialidad a una dimensión física como plantea Castells (1978), donde expone que "el espacio es algo bien material indispensable para la actividad humana" (p.485). No obstante, el espacio es el lugar físico donde se organizan prácticas sociales, pero también lo que significan culturalmente, lo que Aguado y Portal (1991) definen como "la red de vínculos de significación que se establece al interior de los grupos, con las personas y las cosas” (p.37).

Con base en los estudios desarrollados por la geografía humanista y emocional, Tuan (1979), menciona que el estudio del espacio considera los sentimientos espaciales y las ideologías de un grupo a través de las experiencias vividas personales considerada como espacio personal y espacio grupal, donde se es capaz de vivir la experiencia del otro. Es decir, la conceptualización de espacio varía según el contexto, sociedad y cultura a la que se pertenece.

En síntesis, cualquier actividad o acción humana está condicionada por un espacio determinado que componen el "territorio y lugar, símbolos y huellas de la condición y relaciones sociales de quienes lo habitan" (Viñao, 1992, p. 19). Por un lado, está el espacio vivido como determinante en la conformación de la personalidad y mentalidad del sujeto y de una comunidad. Por otro lado, el espacio como un territorio, lugar o paisaje que puede ser visto desde la dimensión física, o como un significado y construcción social, según las experiencias vividas en ese espacio determinado (Viñao, 1992). En este sentido, la escuela se vuelve un espacio que tiene como finalidad aprender los saberes y conocimientos, que se mantiene en constante cambio con el fin de adaptarse a todos y todas las estudiantes y al cuerpo de docente que la componen.

La escuela es espacio y lugar, una institución física y material y, así también, una construcción social y cultural (Viñao, 1992). Al respecto, el ambiente también es parte del espacio al que pertenece la escuela, entendiendo que este se refiere al "contexto y todo aquello que rodea al hombre, lo que puede influenciarlo y puede ser influenciado por él” (Castro y Morales, 2015, p. 3). Es decir, el espacio también es influenciable según el contexto, sociedad y cultura al que pertenecen los y las estudiantes, de manera que, si la escuela quiere utilizar el espacio como herramienta de regulación emocional o como facilitador en el proceso de formación 
del grupo estudiantil, debe contextualizar los contenidos, según los saberes y conocimientos que poseen todos y todas las estudiantes y las características socioculturales en que se formaron. Por consiguiente, la espacialidad desde la construcción conceptual y formación indígena es diferente a la construcción conceptual del espacio desde la mirada occidental de las sociedades dominantes.

\section{ConClusión}

En la actualidad, la escuela es un espacio para la transmisión de conocimientos y saberes tanto conceptual, procedimental y actitudinal. Sin embargo, para que esto se lleve a cabo, el alumnado debe desarrollar el sentido de pertenencia a dicho espacio que abarca más allá de la dimensión física, sino también comprende la dimensión natural, social, cultural y espiritual. Asimismo, para que se comprenda la espacialidad como una dimensión multidimensional y su influencia en la regulación emocional, surge la necesidad de que la educación escolar considere los conocimientos y saberes mapuche para el desarrollo integral de todos los y las estudiantes contribuyendo en el desarrollo de una educación contextualizada a la diversidad social y cultural presente en el aula. Puesto que, la sociedad y cultura a la que pertenecen se hace fundamental en las experiencias vividas que pueden influir en el desarrollo de creencias, valores y principios que están directamente relacionados con la dimensión emocional y afectiva.

En el marco de la educación escolar chilena, históricamente se ha desvalorizado los saberes y conocimientos mapuche, lo que les ha obligado a adoptar características de las sociedades eurocéntricas occidentales, influyendo negativamente en el sentido de pertenencia e identidad, lo que afecta en la autorregulación emocional afectiva. Al respecto, para que los niños y niñas desarrollen la capacidad de regulación emocional, se debe mantener un equilibrio físico, espiritual y con el medio natural. El desequilibrio de estas se presenta en el plano físico o psicológico como un kutran (enfermedad).

Las diferentes ciencias han identificado y catalogado el espacio, la geografía tradicional desde la mirada física, la psicología desde lo personal, la geografía humanista ha integrado el aspecto emocional y el sentido que se le da a los territorios o lugares y la cosmovisión mapuche que ha incorporado a todo lo anterior las fuerzas de carácter espiritual que permanecen en los distintos espacios. Lo anterior, permite identificar las diferencias epistemológicas que se han desarrollado según los contextos socioculturales y según las investigaciones que han aportado las ciencias. Estos antecedentes exponen la diversidad de contenidos que surgen según el contexto en el que se estudian los diferentes elementos. Por consiguiente, se hace necesario el diálogo no solo de parte de las ciencias, más bien, un dialogo entre sociedades y culturas diferentes para el enriquecimiento $\mathrm{y}$ aporte al conocimiento desde diversos enfoques.

La evidencia ha dejado en manifiesto que existen diversos factores influyentes en la capacidad de regular las emociones en el contexto escolar, tales como la familia, el cuerpo de profesores, compañeros, compañeras o pares, equipos de gestión de la escuela, ambiente y contexto. A esta agrupación de elementos, se agrega la dimensión espacial, no vista desde la episteme occidental y reduccionista, sino desde la complejidad de elementos que la conforman; aspecto natural, aspecto sociocultural y espiritual.

Por último, la educación escolar se encuentra en un desafío, que consiste en considerar los saberes indígenas en las políticas educativas que contribuyan a la construcción de un diálogo de saberes socioculturales, que terminen con la descontextualización de los conocimientos basados en un proceso de enseñanza y aprendizaje homogeneizante con características eurocéntricas occidentales. Más bien, la escuela tiene la necesidad de convertirse en un espacio donde se eduque emocionalmente de manera contextualizada y pertinente a sus estudiantes. Para esto, se debe escuchar también los ideales emocionales-afectivos y los conocimientos que poseen todos y todas las estudiantes pertenecientes a grupos minoritarios. 


\section{REFERENCIAS}

Aguado, J., y Portal, M. (1991). Tiempo, espacio e identidad social. Alteridades, 2(1), pp.31-41. Recuperado de https ://alteridades.izt.uam.mx/index.php/Alte/article/view/667/664

Boyd, B., Dooley, K., y Odom, S. (2006). Measuring Learning In The Affective Domain Using Reflective Writing About A Virtual International Agriculture Experience. Journal of Agricultural Education, 3(47), 24-32 Recuperado de https://bit.ly/2RHp5lX

Calderón, E. (2017). El papel de la dimensión afectiva en la adquisición del lenguaje materno. Alteridades, 27(53), pp.11-22. Recuperado de https://alteridades.izt.uam.mx/index.php/Alte/article/view/864

Castro, M., y Morales, M. (2015). Los ambientes de aula que promueven el aprendizaje, desde la perspectiva de los niños y niñas escolares. Revista Electrónica Educare, 19(3), 1-32. doi: https://doi.org/10.15359/ree.19-3.11

Castells, M. (1978). La cuestión urbana. México: Siglo XXI. Recuperado de https://bit.ly/2PI3aIH

Echavarría, C. (2003). La escuela: un escenario de formación y socialización para la construcción de identidad moral. Revista Latinoamericana de Ciencias Sociales, Niñez y Juventud, 1(2), pp.15-43. Recuperado de https://bit.ly/ $36 \mathrm{sHffi}$

Errázuriz, G. (2006). El Pueblo Mapuche: Historia, medicina y proyectos de coexistencia en el área de la salud (Primera parte).Revista Chilena de Pediatría, 77(3),290-294. doi: https://doi.org/10.4067/S0370-41062006000300010

García, R., Ortega, N., y Rivera, A. (2013). El papel de la familia en el aprendizaje compartido de la regulación emocional como bienestar subjetivo. European Scientific Journal, 9(32), 153-161. Recuperado de http://eujour nal.org/index.php/esj/article/view/2020/1932

Henao, G., y García, M. (2009). Interacción familiar y desarrollo emocional en niños y niñas. Revista Latinoamericana de Ciencias Sociales, Niñez y Juventud, 7(2), 785-802. Recuperado de https://bit.ly/35ifBkV

Huerta, A. (2017). El sentido de pertenencia y la identidad como determinante de la conducta, una perspectiva desde el pensamiento complejo. REDIECH, 9(16), 83-97. Recuperado de https://www.redalyc.org/jatsRepo/5216/5 21654339005/521654339005.pdf

Instituto Nacional de Estadísticas. [INE]. (2017). Síntesis de resultados, Censo 2017. Santiago. Chile: Instituto Nacional de Estadísticas. Recuperado de https://bit.ly/3cc2oha

Losada, F. (2001). El espacio vivido. Una aproximación Semiótica. Cuadernos de la Facultad de Humanidadesy Ciencias Sociales, 17, pp.271-294. Recuperado de https://www.redalyc.org/pdf/185/18501716.pdf

Lotito, F. (2009). Arquitectura, psicología, espacio e individuo. AUS, 6, 12-17. Recuperado de http://revistas.uach.c 1/pdf/aus/n6/art03.pdf

Márquez-Cervantes, M., y Gaeta-González, M. (2017). Desarrollo de competencias emocionales en pre-adolescentes: el papel de padres y docentes. Revista Electrónica Interuniversitaria de Formación del Profesorado, 20(2), 221-235. doi: https://doi.org/10.6018/reifop/20.2.232941

Medrano, L., Moretti, L., Ortiz, Á. y Pereno, G. (2013). Validación del Cuestionario de Regulación Emocional Cognitiva en Universitarios de Córdoba, Argentina. Psykhe, 22(1), p.83-96. doi: http://dx.doi.org/10.7764/p sykhe.22.1.473

Nogué, J. (2014). Sentido del lugar, paisaje y conflicto. Geopolitica(s), 5(2), 155-163. doi: https://doi.org/10.5209/r ev_GEOP.2014.v5.n2.48842

Nogué, J. (2015). Emoción, lugar y paisaje. En A. L. Isabel Valverde, Teoría y paisaje II: Paisaje y emoción. El resurgir de las geografias emocionales (p. 170). Barcelona: Universidat Pompeu Fabra. Recuperado de http://www.catpa isatge.net/fitxers/publicacions/teoria_paisaje2/tp_8.pdf

Quintriqueo, S. y Torres, H. (2013). Construcción de conocimiento mapuche y su relación con el conocimiento escolar. Estudios Pedagógicos, 39(1), pp.199-216. doi: https://doi.org/10.4067/S0718-07052013000100012

Quintriqueo, S., Quilaqueo, D., Gutiérrez, M., Peña-Cortés, F. (2015). Enseñanza de Historia, Geografía y Ciencias Sociales: hacia una Perspectiva Intercultural. Temuco. Universidad Católica de Temuco. Recuperado de http:/ /inatuzugumew.uct.cl/wp-content/uploads/2019/03/ciencias-sociales.pdf 
Riquelme, E., Quilaqueo, D., Quintriqueo, S., y Loncón, E. (2016). Predominancia de la educación emocional occidental en contexto indígena: necesidad de una educación culturalmente pertinente. Psicología Escolar e Educacional, 20(3), 523-532. doi: https://doi.org/10.1590/2175-3539201502031038

Ruiz, J. (1994). El espacio escolar. Revista Complutense de Educación, 5(2), 93-104. Recuperado de https://revistas.u cm.es/index.php/RCED/article/view/RCED9494220093A

Saavedra, A. (2000). Notas sobre la población mapuche actual. Revista Austral de Ciencias Sociales, 4, 5-26. Recuperado de https://dialnet.unirioja.es/servlet/articulo?codigo $=2143969$

Sancho, D. y Martínez, A. (2011). Afectividad positiva y salud. Enfermería Global. 10(24), 120-124. doi: https://do i.org/10.4321/S1695-61412011000400010

Thompson, R. (1994). Emotion Regulation: A Theme in Search of Definition. Monographs of the Society for Research in Child Development, 59(2-3), 25-52. doi: https://doi.org/10.2307/1166137

Torres, H. y Quilaqueo, D. (2011). Conceptos de tiempo y espacio entre los mapuches: racionalidad educativa. Papeles de Trabajo. Centro de Estudios Interdisciplinarios en etnolingüistica y antropología, 22, 13-27. Recuperado de $\mathrm{h}$ ttps://bit.ly/2PdRNck

Tuan, Y. (1979). Space and Place: Humanistic Perspective. En S. Gale, y G. Olsson. (Ed.), Philosophy in Geography. Theory and Decision Library (pp. 387-427). Dordrecht: Springer. doi: https://doi.org/10.1007/978-94-009-93 94-5_19

Vargas, G. (2012). Espacio y territorio en el análisis geográfico, Reflexiones, 91(1) 313-326. Recuperado de https://w ww.redalyc.org/pdf/729/72923937025.pdf

Vázquez, E. y Enríquez, M. (2012). Una aproximación sociocultural a las formas de regulación emocional en cuidadores familiares de enfermos crónicos en Guadalajara, Jalisco. Desacatos, 39, 57-72. Recuperado de https://bit.ly/2E $\operatorname{cg} 507$

Viñao, A. (1992). Del espacio escolar y la escuela como lugar: Propuestas y cuestiones. Historia de la Educación. Revista interuniversitaria, 12-13, pp.17-74. Recuperado de https://bit.ly/2Y07k2b

Weber, M. (1977). Economía y Sociedad. España: Fondo de Cultura Económica. Recuperado de https://bit.ly/35gS W8G

Wright, P. (2003). Colonización del espacio, la palabra y el cuerpo en el Chaco argentino. Horizontes Antropológicos, 9(19), 137-152. doi: https://doi.org/10.1590/S0104-71832003000100006

\section{BY-NC-ND}

